\title{
PATTERNS AND RULES FOR BEHAVIOURAL SUBTYPING
}

\author{
Heike Wehrheim \\ Universitat Oldenburg \\ Fachbereich Informatik \\ Postfach 2503, D-26111 Oldenburg, Germany \\ wehrheim@informatik.uni-oldenburg.de
}

\begin{abstract}
Subtyping relations for object-oriented formalisms describe relationships between super- and subclasses which satisfy the substitutability requirement imposed on types and their subtypes. Behavioural subtyping is concerned with subtypes for active classes with an explicit dynamic behaviour, specifiable for instance by object-oriented formal methods combining state-based with behavioural formalisms.

In this paper we develop syntactic patterns and semantic rules for the statebased part of a subclass which guarantee that the subclass is a behavioural subtype of its superclass. This allows to check for subtypes without computing the behavioural semantics of the class at all. Our results are similar to the ones linking data refinement in state-based methods with failure-divergence refinement in CSP. In contrast to classical data refinement, subtyping has to cope with additional new operations in the subclass.
\end{abstract}

Keywords: Object-orientation, refinement, behavioural subtyping, Z, CSP.

\section{INTRODUCTION}

Inheritance is one of the key features for the success of object-oriented analysis and design methods as well as programming languages. Inheritance is employed to re-use already existing class descriptions and extend them with additional attributes and methods, thus creating a new subclass from an existing superclass. Subtyping for object-oriented formalisms $[1,6,12]$ studies relationships between super- and subclasses with the aim of clarifying when a subclass can be said to be type substitutable for its superclass. Type substitutability means that "a subtype instance can be used wherever a supertype instance is expected" [17]. Subtyping is also studied in the context of active classes with a behavioural semantics (as for instance obtained when using LOTOS or CSP as a specification language). Active classes have an explicit 
specification of the dynamic behaviour which describes synchronisation constraints of the class or certain protocols which have to be obeyed. In this paper, we use the term behavioural subtyping to refer to subtyping for this type of classes. A number of proposals for behavioural subtyping relations have been made (among others [16, 2, 4, 13]; see conclusion for a discussion of related work), with for instance Petri nets or labelled transition systems as semantic basis. The starting point of this paper are three subtyping relations from [9] with proven substitutability properties, two of them also adequate for shared mutable objects. The formal basis for these two relations are transition systems and their (CSP) failures and divergences.

Here we will be concerned with checking subtype relationships among classes specified in object-oriented formal methods combining state-based with behavioural aspects. The formal method which we have in mind (and which we use throughout our examples) is CSP-OZ [7], a combination of CSP [10] and Object-Z [15], but the theory we develop will be independent of this particular choice. Two aspects of CSP-OZ are however of importance: first, the fact that the state-based part of CSP-OZ has a behavioural semantics (and thus we have to use behavioural subtyping relations), and second, that the semantics of the combination is obtained by separately computing the semantics of the Object-Z and CSP part, and afterwards joining them by CSP parallel composition. This second point allows us to look at state-based and behaviour part in isolation when checking for subtypes.

A straightforward approach to check subtyping relationships among classes is the following: given two class descriptions, compute their behavioural semantics and compare the classes according to some chosen relation. However, since the semantics of CSP-OZ (and most other such types of integrated formal methods) has to be constructed by hand, this approach is in general impracticable. Instead we are interested in developing simple, at the best syntactic, patterns, which allow to avoid the explicit check and instead guarantee subtypes by construction. Such patterns or rules have to give conditions on attributes and operations in subclasses, which, when followed, immediately lead to subtypes. In this paper we will solely be interested in subtyping patterns for the state-based part (which, due to the compositional semantics, can without change be combined with patterns for the behaviour part). Our investigations are similar to the work about the relationship between data refinement rules in state-based formalisms and failure-divergence refinement $[11,18]$. There, it has been studied whether data refinement induces failuredivergence refinement when the state-based formalism is given a behavioural semantics. The basic approach taken here is the same: we assume to have a behavioural semantics for a state-based formalism, have a set of conditions on two class specifications and show that the conditions induce a behavioural subtyping relationship between the classes (soundness of rules). 
We illustrate our approach by a small example of a buffer which is extended by means of inheritance in three different ways yielding three different kinds of subtypes.

\section{CLASSES}

We start with the specification of the buffer. We use CSP-OZ [7] syntax in the specifications. The following class specifications will not contain any CSP behaviour descriptions since we are only interested in patterns for the statebased part. Thus the classes almost look like Object-Z classes. The specified buffer can hold elements (in a finite bag) of a basic type Element up to some arbitrary number $n$. Elements may be put into the buffer and retrieved by means of the methods put and get, and an attribute alive is used to indicate whether some specific instance of class Buffer is alive or has already been deleted.

\section{[Element $]$}

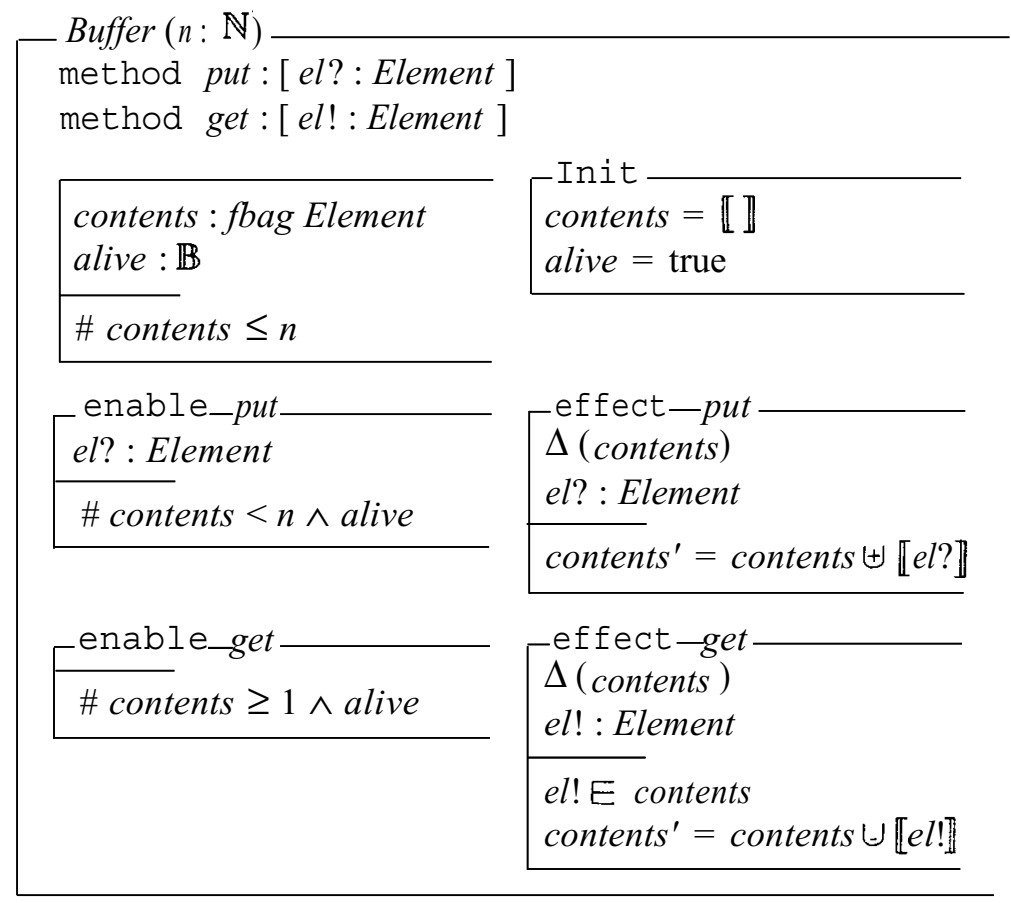

The class is parameterised in the length of the buffer. The first two lines define the interface of the class: it has methods put and get. Next the attributes and their initial values are defined. The application of methods put and get is described by enable and effect predicates, the enabling predicate specifying a guard for the application of the method, the effect predicate the possible results of applying the method. 
In general we represent a class $C$ by a state schema, an initialisation schema and a number of operation schemas: $C=\left(\text { State, Init, (enable_O } O p_{j}\right)_{j \in J \text {, }}$ $\left.\left(\text { effect_} O O p_{j}\right)_{j \in J}\right)$. The components have the following meaning: State describes the state space of the class. It is determined by giving a set of attributes Attr and their types together with an invariant Inv further restricting the allowed values. Init describes the set of initial states determined by a predicate over Attr. $\left(O p_{j}\right)_{j \in J}$ gives a family of methods or operations on the state space. Every operation $O p_{j}$ has a number of input variables $\left(i n_{j, i}\right.$ ? $) \quad 1 \leq i \leq k j$ of type $T_{i n_{j, i}}$ and a number of output variables $\left(\text { out }_{j, i} !\right)_{1 \leq i \leq l_{j}}$ of type $T_{\text {out } j, i}$. Two schemas are given for every operation (an omitted schema in the CSP-OZ specification corresponds to true):

1 an enabling schema enable_op $p_{j}$ whose predicate ranges over the current state Attr and input variables $i_{j, i}$ ?. It acts as a guard to the operation execution, and

2 an effect schema effect_o $o p_{j}$ with predicate ranging over current state $A t t r$, next state $A t t r^{\prime}$, input variables $i n_{j, i}$ ? and output variables $o u t_{j, i}$ ! which describes the postcondition of the operation (where Attr' refers to the new state of attributes after execution of the operation).

We use the following abbreviation for input and output variables:

$$
\begin{aligned}
& I n_{j} \widehat{=}\left[i n_{j, 1} ?: T_{i n_{j, 1}} ; \ldots ; i n_{j, k_{j}} ?: T_{i n_{j, k_{j}}}\right]
\end{aligned}
$$

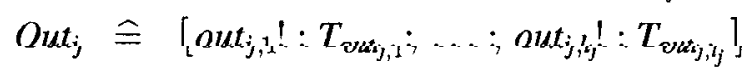

Additionally we define the precondition of an operation $O p_{j}$ to be pre_Op $p_{j}:=$ $\exists$ State', Out $_{j}$ : effect_Op $p_{j}$.

Next we provide the class description with a behavioural semantics in terms of labelled transition systems. The labels are taken from an alphabet $\sum$ which contains all events op. $i_{1} \ldots i_{k} . o_{1} \ldots o_{l}$ where $o p$ is the name of a method and the rest are possible values of input and output parameters. For an event $e v=$ $o p . i_{1} \ldots i_{k} . o 1_{1} \ldots o_{l}$ we write $c h(e v)=o p$ to refer to the method or operation name of an event. $\Sigma_{\tau}$ stands for $\Sigma \cup\{\tau\}$. A labelled transition system (LTS) is a tuple $T=\left(Q, \rightarrow, Q^{i n}\right)$ with $Q$ a set of states, $\rightarrow$ a transition relation and $Q^{i n}$ the set of initial states. A state $q$ is said to be stable if it has no outgoing $\tau$ transitions, $q$ diverges $(q \uparrow)$ if an infinite number of $\tau$ steps is possible from $q$, and $\operatorname{next}(q)$ is the set of all events which are executable from $q$.

The basic idea of the semantics is to view a class as a process which is continuously executing enabled operations and thus changes its state. Initially it is in one of the states of Init. The symbol $\perp$ is used to indicate one particular divergent state: The class diverges if an enabled operation is applied outside its precondition. In the following we take a schema to stand for a set of bindings and for a binding $q=\backslash a_{1} \rightrightarrows v_{1}, \ldots, a_{n} \Rightarrow v_{n} \downarrow$ and a schema $S$ we write $S(q)$ 
if $q$ is a valid binding of schema $S$. The operator $\cup$ is used to join bindings: $\backslash a_{1} \Rightarrow v_{1}, \ldots, a_{n} \Rightarrow v_{n} \backslash \cup \vee b_{1} \Rightarrow w_{1}, \ldots, b_{m} \Rightarrow w_{m} \downarrow=\backslash a_{1} \Rightarrow$ $v_{1}, \ldots, a_{n} \Rightarrow v_{n}, b_{1} \Rightarrow w_{1}, \ldots, b_{m} \Rightarrow w_{m} D$ assuming disjointness of the sets $\left\{a_{1}, \ldots, a_{n}\right\}$ and $\left\{b_{1}, \ldots, b_{m}\right\}$. For a state $q$, the notation $q$ ' is used to describe the same binding but with components decorated with ', the notation $\left.q\right|_{\text {Sate }}$ is a binding which only gives values to variables in $q$ which are declared in State.

\section{Definition 2.1}

The operational semantics of a class $C$ is defined as $\llbracket C \rrbracket=\left(Q_{C}, \rightarrow_{C}, Q_{C}^{i n}\right)$, where

- $Q_{C}=$ State $\cup\{\perp\}, Q_{C}^{\text {in }}=$ Init and

- $\rightarrow_{C}$ is defined as $\perp \stackrel{\tau}{\rightarrow}_{C} \perp$ and

$$
\begin{aligned}
& -q_{1} \stackrel{e v}{\longrightarrow} C q_{2} \text { if } e v=o p_{j} . i_{1} \ldots . i_{k_{j}} . \text { out }_{1} \ldots \text {.out } t_{j} \text { and } \\
& \text { enable_op } p_{j}\left(q_{1} \cup \backslash i n_{n} \Rightarrow i_{n}, 1 \leq n \leq k_{j} \downarrow\right) \text { and } \\
& \text { effect_op } p_{j}\left(q_{1} \cup q_{2} \cup \cup i n_{n} \Rightarrow i_{n}, o u t_{m} \Rightarrow o_{m}, 1 \leq n \leq k_{j}, 1 \leq\right. \\
& \left.m \leq l_{j} D\right) \text {, } \\
& -q \stackrel{e v}{\rightarrow}+\text { if ev }=o p_{j} . i_{1} \ldots i_{k_{j}} . o u t_{1} \ldots \text { out }_{l_{j}} \text { and }
\end{aligned}
$$

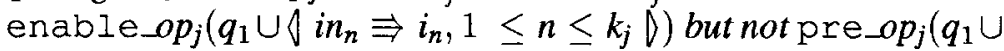

$$
\begin{aligned}
& \left.\left.\checkmark i n_{n} \Rightarrow i_{n}, 1 \leq n \leq k_{j}\right\rangle\right) \text {. }
\end{aligned}
$$

Note that we use state $q_{2}$, here since the effect predicate uses primed variables to refer to the state after execution.

The dynamic behaviour of class Buffer(2) with an instantiation of type Element with the set $\{1,2\}$ is depicted by the transition system in Figure 1 .

Next we present three extensions of the basic class Buffer by inheritance: the first adds a possibility of explicitly deleting the buffer, the second adds a new method checking for emptiness and the third a method which allows to insert two elements at once.

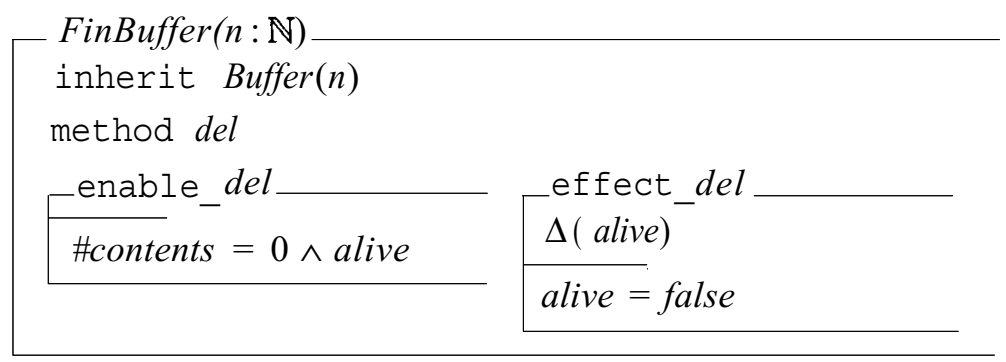




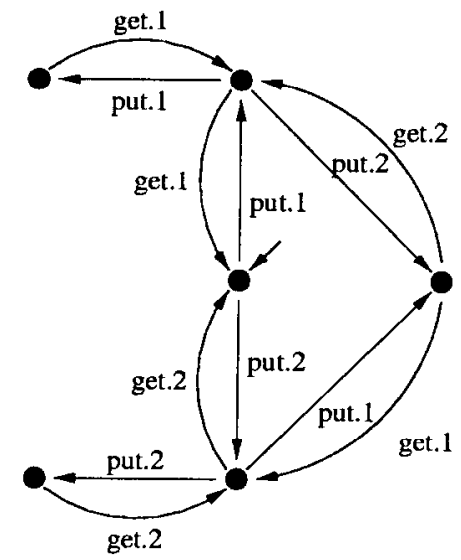

Figure 1. Dynamic behaviour of superclass Buffer (2)
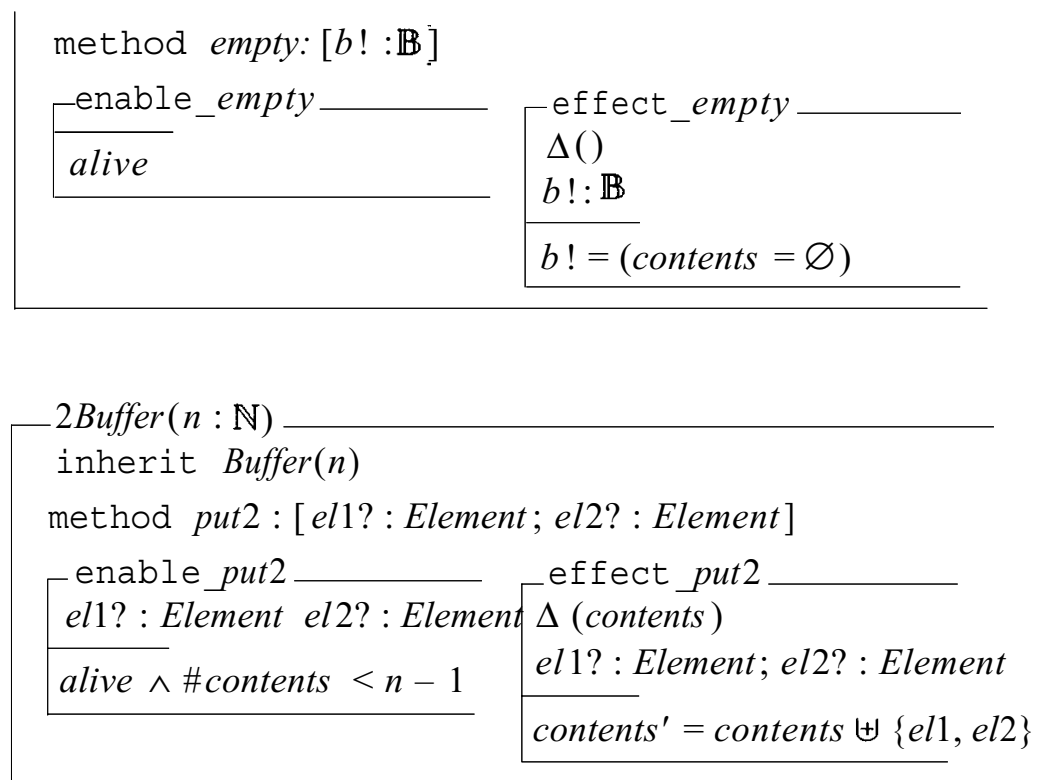

Conceptually inheritance is defined as conjunction on schemas: given an abstract class $A=\left(\right.$ S State, AInit, $\left.\left(\text { enable } A O p_{j}\right)_{j \in J},\left(\text { effect } A O p_{j}\right)_{j \in J}\right)$ and specialised class $C=\left(\right.$ CState, CInit, $\left.(\text { enable_COp })_{i \in I},\left(\text { effect_COp } p_{j}\right)_{j \in J}\right)$ assuming $C$ is constructed from $A$ by means of inheritance, then $J \subseteq I$ and

$$
\begin{aligned}
\text { CState } & =\text { AState } \wedge \text { ExtState } \\
\text { Clnit } & =\text { AInit } \wedge \text { ExtInit },
\end{aligned}
$$




$$
\begin{aligned}
& \text { enable_COp } p_{j}=\text { enable_AOp } p_{j} \Lambda \text { enable_ExtOp } p_{j} \quad \forall j \in J \text {, } \\
& \text { effect_COp } p_{j}=\text { effect_AOp } p_{j} \Lambda \text { effect_ExtOp } p_{j} \quad \forall j \in J \text {, } \\
& \text { enable_COp } p_{i}=\text { enable_ExtOp } p_{i} \quad \forall i \in I \backslash J, \\
& \text { effect_COp } p_{i}=\text { effect_ExtOp } p_{i} \quad \forall i \in I \backslash J .
\end{aligned}
$$

ExtState (ExtInit etc.) is the schema which is explicitly written in the subclass and AState (etc.) is inherited.

\section{SUBTYPING}

Subtyping on ordinary types is usually demanded to fulfill the requirement of type substitutability: subtypes should be usable wherever supertypes are expected without any noticeable differences [17]. When lifting this concept to classes, the first idea is to use the inheritance hierarchy for defining subtyping relations. This however does not fit the requirement of substitutivity anymore: inheritance is first of all concerned with re-use of class descriptions and allows fundamental changes to the behaviour of superclasses in a subclass. The requirement of substitutivity suggests that instead some sort of refinement should be used as a subtyping relation,

Since we are working in the context of CSP, our notion of refinement is failure-divergence refinement. In the process algebra CSP process refinement is used to compare an implementation against a specification with respect to their failures and divergences: the implementation has to be more deterministic than the specification, i.e. has less failures and divergences. Failures are sets of pairs $(\sigma, X) \in \Sigma^{*} \times 2^{\Sigma}$ with the meaning that a process has such a pair in its failure set if it can execute the trace $\sigma$ and may thereby reach a stable state (no $\tau$-transitions possible) in which none of the events from $X$ are possible. Divergences are sets of traces $\sigma$ such that a process may diverge after the trace, i.e. execute an infinite number of invisible actions. Failures and divergences can for instance be computed from the transition system of a process, but due to lack of space we refrain from giving a formal definition here. Buffer(2) for instance has failures (with maximal refusals) $(\varepsilon,\{$ get. 1, get. 2$\}),($ put $.1,\{$ get .2$\}),($ put. $1 \cap$ put .2, \{put.1, put .2\}), ... and an empty set of divergences.

Refinement then simply is inclusion of failure and divergence sets: A labelled transition system $I$ is a failure-divergence refinement of an 1 ts $S(S \sqsubseteq \mathcal{F D}$ $I)$ iff failures $(I) \subseteq$ failures $(S)$ and divergences $(I) \subseteq$ divergences $(S)$.

According to this definition none of the subclasses of Buffer are refinements (e.g. FinBuffer has a failure (del, $\Sigma$ ) which is not in Buffer's failure set). Nevertheless refinement will be the basis for our three behavioural subtyping relations. The relations additionally have to cope with the fact that the subclass may introduce new methods. The comparison of sub- with superclass has to 
"ignore" these methods or at least relate new methods to old ones. For this, we use three different operators: restriction, concealment (next) and substitution (below), and define them by their effect on failures and divergences.

Definition 3.1 Let $T=\left(Q, \rightarrow, Q^{\text {in }}\right)$ be a labelled transition system and $N \subseteq$ ¿. Restriction: failures $\left(T \backslash_{r} N\right)=\{(\sigma, X) \mid \exists Y \bullet(\sigma, Y) \in$ failures $(T), \sigma \in$ $\left.(\Sigma \backslash N)^{*}, X \subseteq Y \cup N\right\}$,

- Concealment: failures $\left(T \backslash_{c} N\right)=\left\{(\sigma, X) \mid \exists\left(\sigma^{\prime}, Y\right) \in\right.$ failures $(T), \sigma=$ $\left.\sigma^{\prime} \mid(\Sigma \backslash N) \wedge X \subseteq Y \cup N\right\}$.

Divergences are unchanged by restriction and concealment.

Restriction forbids the execution of events in $N$, concealment makes $N$-events invisible. Note that concealment differs from classical hiding in process algebras. Hiding may usually introduce new non-stable states, whereas concealment does not.

The basic idea of substitution is to map new methods onto sequences of old methods: the state changes achieved by new method should be the same as that achieved by a consecutive execution of the old methods. This is the "dynamic behaviour" counterpart of Liskov and Wing's extension maps [12].

Definition 3.2 Let $(\sigma, X) \subseteq \Sigma \times \mathbf{2}^{\Sigma}$ be a pair of trace and refusal, and $N \subseteq \Sigma$ a set of events (the new events to be mapped onto old ones). Furthermore let $f_{N}: N \rightarrow \Sigma^{*}$ be a function, the substitution function. We define $f_{N}(\sigma, X)$ (by overloading the function $\left.f_{N}\right)$ to be $\left(f_{N}(\sigma), X \cup N\right)$ where $f_{N}(\sigma)$ is inductively defined by $f_{N}(\varepsilon)=\varepsilon$ and

$$
f_{N}(a \frown \sigma)= \begin{cases}a^{\frown} f_{N}(\sigma) & \text { if } a \notin N \\ f_{N}(a) \frown f_{N}(\sigma) & \text { else }\end{cases}
$$

Superclass and subclass are then compared by applying concealment, restriction or substitution to the new methods before checking for failure and divergence inclusion (see [9]).

Definition 3.3 Let A, C be labelled transition systems, A standing for the superand $C$ for the subclass, and let $N$ be the set of events over new methods.

- $C$ is a weak subtype of $A$ (denoted $A \sqsubseteq_{w s t}^{N} C$ ) iff $A \sqsubseteq \mathcal{F D} C \backslash_{r} N$.

- $C$ is a safe subtype of $A$ according to a substitution function $f_{N}$ (denoted $\left.A \sum_{s s t}^{f_{N}} C\right)$ iff $\forall(\sigma, X) \in$ failures $(C): f_{N}(\sigma, X) \in$ failures $(A)$ and $\forall \sigma \in$ divergences $(C): f_{N}(\sigma) \in$ divergences $(A)$.

- $C$ is an optimal subtype of $A$ (denoted $\left.A \sqsubseteq_{\text {ost }}^{N} C\right)$ iff $A \sqsubseteq \mathcal{F D} C \backslash_{c} N$. 
Coming back to our four buffers: FinBuffer is a weak subtype of Buffer and EmptyCheckBuffer is a weak as well as an optimal subtype. FinBuffer is not an optimal subtype of Buffer since 【FinBuffer $\rrbracket \backslash_{c}\{$ del $\}$ has a failure $(\varepsilon, \Sigma)$ which Buffer does not have. Finally 2 Buffer is a safe subtype (mapping puf2 to put $\frown$ put) as well as a weak subtype of Buffer but no optimal subtype.

In order to check for subtypes we first had to construct the transition systems, compute failures/divergences for the classes and afterwards apply the subtyping definition. Next we look at patterns which give us the possibility of obtaining a subtype by construction and rules which allow to locally check for subtypes without computing the semantics of classes at all.

\section{PATTERNS AND RULES}

We refer to patterns as conditions on the specification of a subclass which can be checked (almost) completely syntactically, without looking at the semantics at all. Rules will be conditions on classes which can be checked by looking at the semantics of methods in isolation, i.e. without computing the transition system semantics of a class but by comparing the meaning of methods of sub- and superclass.

Besides the patterns and rules discussed here, it is always possible to employ data refinement rules (specific to CSP-OZ [8]): any subclass which is a refinement of its superclass is a subtype in the sense of all three relations. All of the following patterns and rules can thus be combined with a valid data refinement.

Patterns. We present two patterns here, one for weak and one for optimal subtyping. The patterns are conditions on the changes and extensions allowed in the subclass. We again assume $A=\left(\right.$ AState, AInit, $\left.\left(A O p_{j}\right)_{j \in J}\right)$ to be the super- and, letting $J \subseteq I, C=\left(C S t a t e, C I n i t,\left(C O p_{i}\right)_{i \in I}\right)$ to be the subclass.

P1 $\forall j \in J$ : enable_COp $p_{j}=$ enable $A O p_{j}$ and effect_COp $p_{j}=$ effect $A O p_{j}$, i.e. the specialised subclass does not change the old methods at all. There are no restrictions on new methods or attributes.

P2 P2.A $\forall j \in J$ : enable $\_O p_{j}=$ enable $\_A O p_{j}$ and effect_COp $p_{j}=$ effect $A O p_{j}$,

P2.B $\forall n \in I \backslash J$ : the predicate of schema effect_COp $p_{n}$ ranges solely over Attr, ExtAttr and ExtAttr', i.e. no changes to old attributes are allowed,

P2.C $\forall n \in I \backslash J$ : enable_COp $p_{n} \Rightarrow$ pre_COp $p_{n}$.

Here $=$ stands for syntactical equality. Pattern P1 is a pattern for a weak subtype, e.g. FinBuffer may be constructed from Buffer using pattern P1, it leaves 
all old methods unchanged. Pattern P2 is valid for EmptyCheckBuffer: the new method empty does not change old attributes and its precondition holds whenever the method is enabled. We however cannot construct FinBuffer from Buffer using pattern P2 since FinBuffer's effect predicate for operation del changes a value of an old attribute (alive). Condition P2.C is essentially used to avoid new divergences in the subclass. It is the only part of the patterns which is not purely syntactic, but this is necessary since we want to take divergence into account.

The following main theorem now states the soundness of the patterns for the subtyping relations.

\section{Theorem 1}
If $C$ is a subclass of $A$ obeying pattern P1 then $A \sqsubseteq_{\text {wst }} C$. If $C$ is a subclass of $A$ obeying pattern $P 2$ then $A \sqsubseteq_{\text {ost }} C$.

Note that such a relationship cannot be guaranteed by inheritance alone. Inheritance allows all kinds of changes in the subclass. Due to lack of space we only show the proof for the second part of the theorem. For the proof we show that, when constructed by a pattern, subclass and superclass are even more close to each other than defined by subtyping: the superclass simulates the subclass in a certain sense. Hence in the following we define a simulation relation inducing optimal subtyping. The relation is in spirit a forward simulation. We do not consider backward simulations here since we are not interested in completeness. In fact, it is immediately clear that the patterns are not complete: there are as well valid subtypes which cannot be constructed according to the patterns.

For the simulation definition we assume that the semantics of Definition 2.1 is used for classes, with the consequence that there are no $\tau$-transitions except from $\perp$ to $\perp$.

Definition 4.1 Class $A$ simulates $C$ concealing $N, A \preceq_{(N)} C$, if there is a relation $\rho \subseteq Q_{A} \times Q_{C}$ such that

$$
\begin{aligned}
& 1 \forall q_{C} \in Q_{C}^{i n} \exists q_{A} \in Q_{A}^{i n}:\left(q_{A}, q_{C}\right) \in \rho, \\
& 2 \forall\left(q_{A}, q_{C}\right) \in \rho \text { the following holds: } \\
& -\operatorname{stable}\left(q_{C}\right) \Rightarrow \text { stable }\left(q_{A}\right), \operatorname{next}\left(q_{C}\right) \backslash N=\operatorname{next}\left(q_{A}\right), q_{C} \uparrow \Rightarrow q_{A} \uparrow, \\
& -\forall a \notin N: \\
& \quad q_{C} \stackrel{a}{\rightarrow} C q_{C}^{\prime} \Rightarrow \exists q_{A}^{\prime}: q_{A} \stackrel{a}{\rightarrow}_{A} q_{A}^{\prime} \wedge\left(q_{A}^{\prime}, q_{C}^{\prime}\right) \in \rho, \\
& \forall a \in N: \\
& \quad q_{C} \stackrel{a}{\rightarrow}_{C} q_{C}^{\prime} \Rightarrow\left(q_{A}, q_{C}^{\prime}\right) \in \rho .
\end{aligned}
$$

The idea behind simulation for optimal subtypes is that for the $\mathrm{N}$-steps of the subclass we do not require a matching step of the superclass, instead states $q^{\prime}$ 
reached after executing new methods from some state $q$ must be simulated by the matching partner of $q$.

\section{Theorem $2 A \preceq_{(N)} C \Rightarrow A \sqsubseteq_{o s t}^{N} C$.}

The remaining part for proving (the second part of) Theorem 1 is to show that subclasses constructed by patterns can be simulated by the superclasses. We again use $N$ to refer to the set of all events over new methods.

Theorem 3 If $C$ is a subclass of A obeying pattern P2 then $A \preceq_{(N)} C$.

Rules. We proceed with giving rules for the last subtyping relation, safe subtyping. Here, we actually have to compare the semantics of new methods with that of (sequences of) old methods. This requires a little more technical overhead. We explain this by means of 2 Buffer. 2 Buffer is a safe subtype of Buffer since the new method put 2 can be explained in terms of the old method put: the effect of put 2 is the same as that of two put's. However, the substitution function $f_{N}$ that we need does not map a new method/operation to a sequence of operations but events to sequences of events. Besides transformation of operations we also need some means for transforming inputs and outputs of a new operation onto the inputs and outputs of the old operations. For this, we use (an adaption of) the technique of input and output transformers as for instance advocated in [5]. An input or output transformer simply is a schema that relates inputs (or outputs) of operations. Looking at 2 Buffer the input transformer should relate el1? and el2? with the two inputs el? of two put's, the output transformer is empty. In order to be able to distinguish the inputs of the two put's we define another decoration on schemas, that decorate input and output variables with numbers.

For an operation schema $S=\left[a_{1}: T_{1} ; \ldots ; a_{n}: T_{n}\right.$, in? : $T_{\text {in }}$, out! : $\left.T_{\text {out }} \mid p\right]$ we define $S^{k}, k \in \mathbb{N}$, to be $\left[a_{1}: T_{1} ; \ldots ; a_{n}: T_{n}, i n^{k}\right.$ ? : $T_{\text {in }}$, out $t^{k}$ : $T_{\text {out }} \mid p\left[\right.$ in? $_{\text {? }}$ in $^{k}$ ?,out! $/$ out $^{k}$ ! $\left.]\right]$ (and similar for more than one input or output variable). The decoration is used to distinguish variables with the same name coming from operations at different positions in the substituting sequence. The input transformer from put 2 to $p u t^{1} \stackrel{\circ}{9}$ put $^{2}$ can thus be formulated as:

\begin{tabular}{l} 
el 1 : Elwmwnt; el2?: Element \\
el $!:$ Element $; e l^{2} !:$ Element \\
\hline$e l 1 ?=e l^{1} ! \Lambda e l 2 ?=e l^{2} !$ \\
\hline
\end{tabular}

It transforms inputs of put 2 into outputs which can than be fed into $p u t^{1}{ }_{9}^{\circ} p u t^{2}$ (using the $\mathrm{Z}$ pipe operator $>>$ ). Conversely, an output transformer from a sequence of old operations $A O p_{1}^{1}{ }_{9}^{\circ} A O p_{2}^{2}$ to a new operation $C O p$ would take the 


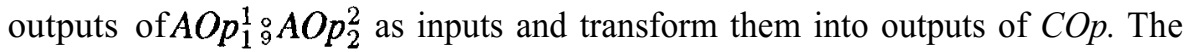
output transformer from puts ${ }_{9}^{1} p^{p} t^{2}$ to $p u t$ is an empty schema: no declarations and the predicate is true.

To simplify matters, we only consider substitutions which map one operation to a sequence of two operations in the sequel. This can however easily be generalised to an arbitrary number of operations.

Definition 4.2 $\mathrm{An}$ input transformer from $\mathrm{COp}$ to $\mathrm{AOP} \mathrm{p}_{1}^{1} \mathrm{O} A O \mathrm{p}_{2}^{2}$ is a schema IT s. $t$.

- all input variables from COp are declared in IT,

- for every input variable $x^{i}$ ? of type $T$ from operation $A O p_{i}^{i}, i=1,2$, there is one output variable $x^{i}$ ! : T in IT,

- no other variables are declared in IT and

- the predicate of IT is a conjunction of equalities of the form $x$ ? = y! such that every output variable occurs exactly once.

Conversely, an output transformer from $A O p_{1}^{1} \stackrel{9}{A} O p_{2}^{2}$ to COp is a schema $O T$ in which all output variables from COp are declared, for every output variable $x^{i}$ ! : T from $A O p_{i}^{i}, i=1,2$, there is one input variable $x^{i}$ ? : T, no other variables are declared in OT, and the predicate of IT is a conjunction of equalities of the form $x$ ? $=y$ ! such that every output variable occurs exactly once.

Figure 2 illustrates the use of input and output transformers: execution of $C O p$ should be the same as that of $A O p_{19} A O p_{2}$, and the input and output transformers define the valid relationships between the input and outputs.

We now again assume $A$ to be the super- and $C$ to be the subclass. The following rule formalises Liskov and Wings's extension maps [12] in our setting.

Extension Rule: The extension rule $R$ holds between $A$ and $C$ if all old methods remain unchanged in the subclass (or are changed according to data refinement rules) and there is an extension map $E: N_{o p} \rightarrow O_{o p}^{*}$ such that the following holds: for every $C O p \in N_{o p}$, either $C O p$ does not change attributes at all and $E(C O p)=\varepsilon$, or else $C O p$ does change attribute values, $E(C O p)=A O p_{1} \frown A O p_{2}$ and there is an input transformer $I T$ from $C O p$ to $A O p_{1}{ }_{9}^{\circ} A O p_{2}$, an output transformer $O T$ from $A O p_{1} \stackrel{\circ}{A} A O p_{2}$ to $C O p$ and the following conditions are furthermore met.

$$
\begin{aligned}
& \text { Rz.A } \forall \text { CState }, \forall I n \bullet \text { enable_COp } \Rightarrow I T \gg\left(\text { enable_AOp }{ }_{1}{ }^{1} \wedge \text { pre_AOp }{ }_{1}{ }^{1}\right) \text {, } \\
& \text { R.B } \forall \text { CState, } \forall I n \bullet \text { enable_COp } \Rightarrow \text { pre.COp, } \\
& \text { R.C } \forall \text { CState, CState }, \forall I n \bullet \text { enable_COp } \wedge\left(I T \gg \text { effect } \_A O p_{1}{ }^{1}\right) \\
& \Rightarrow I T \gg\left(\text { enable_AOp } p_{2}{ }^{2} \wedge \text { pre } A O p_{2}{ }^{2}{ }^{\prime}\right) \text {, }
\end{aligned}
$$




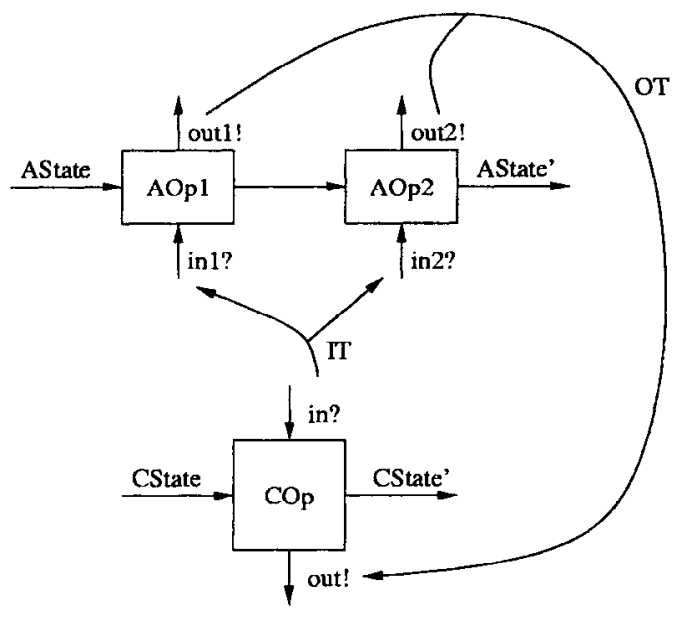

Figure 2. Input and output transformer

\section{R.D $\forall$ CState, CState,$\forall$ In, Out $\bullet$ enable_COp $\wedge$ effect_COp $\Rightarrow I T \gg\left(\right.$ effect $A O p_{1}{ }^{1}{ }_{9}$ effect $\left.A O p_{2}{ }^{2}\right) \gg O T$.}

The conditions have the following meaning: R.A states that whenever $C O P$ is enabled so is $A O p_{1}$ and its precondition is also fulfilled; R.B guarantees that $C O p$ does not introduce new divergences; R.C states that after execution of $A O p_{1}$ the second operation of the sequence $A O p_{2}$ is enabled and defined. Finally R.D guarantees that the effect of executing $C O p$ is the same as that of $A O p_{1}: A O p_{2}$.

An extension map and the corresponding input and output transformers $I T$, $O T$ induce a substitution function $f_{T, O T}^{E}: N \rightarrow \Sigma^{*}$. This function has to transform events into sequences of events; the extension map tells us which operation to map onto which sequence of operations and the input and output transformers tell us the values of parameters. Additionally we need the ordering among inputs and outputs as defined in the interface declaration of the class. Let $C O p$ and $A O p_{1}, A O p_{2}$ be defined by

$$
\begin{aligned}
\text { method } C O P: & {\left[\operatorname{cin}_{1} ?: T_{1}, \ldots, \text { cin }_{k}: T_{k},\right.} \\
& \left.\operatorname{cout}_{1}: T_{k+1}, \ldots, \text { cout }_{l}: T_{k+l}\right] \\
\text { method } A O p_{1}: & {\left[\operatorname{ain}_{1,1} ?: T_{1,1}, \ldots, \text { ain }_{1, k_{1}}: T_{1, k_{1}},\right.} \\
& \text { aout } \left._{1,1}: T_{1, k_{1}+1}, \ldots, \text { aout }_{1, l_{1}}: T_{1, k_{1}+l_{1}}\right] \\
\text { method } A O p_{2}: & {\left[\operatorname{ain}_{2,1} ?: T_{2,1}, \ldots, \text { ain }_{2, k_{2}}: T_{2, k_{2}},\right.} \\
& \text { aout } \left._{2,2}: T_{2, k_{2}+1}, \ldots, \text { aout }_{2, l_{2}}: T_{2, k_{2}+l_{2}}\right]
\end{aligned}
$$

Given this declaration we associate with an input transformer $I T$ two functions $\mathcal{I} \mathcal{T}^{i}, i=1,2$, mapping input values of $C O p$ onto input values of $A O p_{1}, A O p_{2}$, 
respectively.

$$
\mathcal{I T}^{i}\left(i_{1}, \ldots i_{k}\right)=\left(w_{1}, \ldots, w_{k_{i}}\right)
$$

with $w_{j}=i_{m}, 1 \leq j \leq k_{i}, 1 \leq m \leq k$, if $\operatorname{cin}_{m}$ ? $=a i n_{j}^{i}$ ! is an equation in IT. For output transformers $O T$ we have a single function that maps ordered outputs of $A O p_{1}{ }_{9}^{\circ} A O p_{2}$ to outputs of $C O p$.

$$
\mathcal{O} \mathcal{T}\left(v_{1,1}, \ldots v_{1, l_{1}}, v_{2,1}, \ldots, v_{2, l_{2}}\right)=\left(o_{1}, \ldots, o_{l}\right)
$$

with $o_{j}=v_{i, m}$ if cout $_{j} !=$ aout $_{m}^{i}$ ? is an equation in $O T$. Then $f_{I T, o T}^{E}$ is defined to be

$$
\begin{aligned}
& f_{I T, o T}^{E}\left(o p . i_{1} \ldots i_{k} . o_{1} \ldots o_{l}\right)= \\
& E^{1}(o p) \cdot \mathcal{I} \mathcal{T}^{1}\left(i_{1}, \ldots, i_{k}\right) \cdot v_{1} \ldots v_{l_{1}} \frown E^{2}(o p) \cdot \mathcal{I T}^{2}\left(i_{1}, \ldots, i_{k}\right) \cdot v_{l_{1}+1} \ldots v_{l_{1}+l_{2}} \\
& \text { such that } \mathcal{O} \mathcal{T}\left(v_{1}, \ldots, v_{l_{1}}, v_{l_{1}+1}, \ldots, v_{l_{1}+l_{2}}\right)=\left(o_{1}, \ldots, o_{l}\right) .
\end{aligned}
$$

Looking at $2 B u f f e r$ we find that $E$ defined by $E($ put 2$)=$ put $\frown$ put is an extension map; for instance \#contents $<\mathrm{n}-1$ implies \#contents $<n$.

The following Lemma is the key to correctness of the extension rule:

\section{Lemma 4.1}

Let $\boldsymbol{A}=\left(\right.$ AState, AInit, $\left.\left(\text { enable } \_O O p_{j}\right)_{j \in J},\left(\text { effect } A O p_{j}\right)_{j \in J}\right)$ be the superand $C=(\text { CState, CInit, (enable_COp })_{i \in I}$, (effect_COp $\left.)_{i \in I}\right)$ the subclass. Let $E: N_{o p} \rightarrow O_{o p}^{*}$ be an extension map with input and output transformers IT, OT for every new operation. Let $T_{\mathcal{C}}=\llbracket C \rrbracket$ and $T_{A}=\llbracket A \rrbracket$ be the transition systems. Then the following holds:

$$
\begin{aligned}
& \forall q_{1} \in Q_{C}: q_{1} \stackrel{e v}{\longrightarrow} q_{2} \text { with } \operatorname{ch}(e v) \notin N_{o p} \text { a new operation } \\
& \Rightarrow \exists q_{3} \in Q_{A}:\left.\left.q_{1}\right|_{\text {AState }} ^{e v_{1}} \longrightarrow q_{3} \stackrel{e v_{2}}{\longrightarrow} q_{2}\right|_{\text {AState }} \\
& \quad \text { such that } f_{I T, O T}^{E}(e v)=e v_{1} \frown e v_{2} .
\end{aligned}
$$

The proof is again omitted. Analogous to the other subtyping relations, we have a simulation relation which induces safe subtyping.

Definition 4.3 Let $f_{N}: N \rightarrow \Sigma^{*}$ be a substitution function. Class $A$ simulates $C$ changing $N$ by $f_{N}, A \preceq_{f_{N}} C$, if there is a relation $\rho \subseteq Q_{A} \times Q_{C}$ such that

$$
l \forall q_{C} \in Q_{C}^{i n} \exists q_{A} \in Q_{A}^{i n}:\left(q_{A}, q_{C}\right) \in \rho,
$$

$2 \forall\left(q_{A}, q_{C}\right) \in \rho$ the following holds:

$$
\begin{aligned}
& \text { - } \operatorname{stable}\left(q_{C}\right) \Rightarrow \text { stable }\left(q_{A}\right), \operatorname{next}\left(q_{C}\right) \backslash N=\operatorname{next}\left(q_{A}\right), q_{C} \uparrow \Rightarrow q_{A} \uparrow, \\
& -\forall a \notin N: \\
& \quad q_{C} \stackrel{a}{\rightarrow}_{C} q_{C}^{\prime} \Rightarrow \exists q_{A}^{\prime}: q_{A} \stackrel{a}{\rightarrow}_{A} q_{A}^{\prime} \wedge\left(q_{A}^{\prime}, q_{C}^{\prime}\right) \in \rho . \\
& \forall a \in N: \\
& \quad q_{C} \stackrel{a}{\rightarrow}_{C} q_{C}^{\prime} \Rightarrow \exists q_{A}^{\prime}: q_{A} \stackrel{f(a)}{\rightarrow} q_{A}^{\prime} \wedge\left(q_{A}^{\prime}, q_{C}^{\prime}\right) \in \rho .
\end{aligned}
$$


As before we get two results relating simulation with subtyping and patterns with simulation.

Theorem $4 A \preceq_{f_{N}} C \Rightarrow A \sqsubseteq_{s s t}^{f_{N}} C$.

If $C$ is a subclass of A obeying the extension rule $R$ then $A \preceq f_{N} C$.

\section{DISCUSSION}

In this paper we presented subtyping patterns and rules for active classes with a behavioural semantics. Patterns are simple, mostly syntactic conditions which, when applied during construction of subclasses via inheritance, guarantee correct subtypes. Rules are conditions which can be locally checked on the semantics of operations.

Related work. First of all there is a huge amount of work on behavioural subtyping relations for object-oriented formalisms. They differ in the semantic models used as a basis and in the defined relations. A rough classification could divide them into bisimulation and refinement-based approaches. Bisimulation based approaches easily achieve some form of substitutivity since they use an equivalence relation for subtyping. We consider this to be too strong since subtyping in its basic nature is not symmetric. Refinement-based proposals on the other hand in most cases only discuss substitutability but do not give a formal proof. The relation most often used in this context is extension, developed for the process algebra LOTOS. Extension allows extension of functionality, but not only on new methods but also on old methods.

The discussion of related work concerning the specific topic of this paper can be split in two parts. The first one concerns the large amount of work on simulation relations for refinement, especially those showing the tight connections between data refinement in state-based and process refinement in behaviour-based systems $[11,18]$. The ideas of these papers are clearly the basis for our simulation relations which adapt this basic concept to the case of behavioural subtyping relations. The second area are patterns for subtyping relations. The work of van der Alst and Basten [16] presents transformation rules on Petri nets which construct subtypes, where the subtyping relations are based on branching bisimilarity. Rudkin [14] studies subtyping patterns for LOTOS, i.e. specific LOTOS operators that induce subtypes. The relation chosen there is again extension.

Patterns which are similar to ours are also employed in UNITY's superposition operator [3]. This is not an object-oriented setting, nevertheless the goal is similar: extend a program in a conservative way, not disturbing any of the properties holding beforehand. In fact, the idea of conservative extension appears in many theories and behavioural subtyping is just a form of conservative extension in object-orientation. 


\section{REFERENCES}

[1] P. America. Designing an object-oriented programming language with behavioural subtyping. In J.W. de Bakker, W.P. de Roever, and G. Rozenberg, editors, REX Workshop: Foundations of Object-Oriented Languages, number 489 in LNCS. Springer, 1991.

[2] H. Bowman, C. Briscoe-Smith, J. Derrick, and B. Strulo. On behavioural subtyping in LOTOS. In H. Bowman and J. Derrick, editors, FMOODS '97, pages 335 - 351. Chapman \& Hall, 1997.

[3] K.M. Chandy and J. Misra. Parallel program design: a foundation. Addison Wesley, 1988.

[4] E. Cusack. Refinement, conformance and inheritance. Formal Aspects of Computing, 3:129 - 141, 1991.

[5] J. Derrick and E. Boiten. Non-atomic refinement in Z. In J. Woodcock and J. Wing, editors, FM'99, number 1709 in LNCS, pages 1477-1496. Springer, 1999.

[6] K. K. Dhara and G. T. Leavens. Forcing behavioral subtyping through specification inheritance. In ICSE '96, pages 258-267. IEEE Computer Society Press, March 1996.

[7] C. Fischer. CSP-OZ: A combination of Object-Z and CSP. In H. Bowman and J. Derrick, editors, FMOODS '97, volume 2, pages 423-438. Chapman \& Hall, 1997.

[8] C. Fischer and S. Hallerstede. Data-Refinement in CSP-OZ. Technical Report TRCF-973, University of Oldenburg, June 1997.

[9] C. Fischer and H. Wehrheim. Behavioural subtyping relations for object-oriented formalisms. In T. Rus, editor, AMAST 2000, number 1816 in LNCS, pages 469-483. Springer, 2000.

[10] C. A. R. Hoare. Communicating Sequential Processes. Prentice-Hall, 1985.

[11] M.B. Josephs. A state-based approach to communicating processes. Distributed Computing, 3:9-18, 1988.

[12] B. Liskov and J. Wing. A behavioural notion of subtyping. ACM Transactions on Programming Languages and Systems, 16(6):1811 - 1841, 1994.

[13] O. Nierstrasz. Regular types for active objects. In O. Nierstrasz and D. Tsichritzis, editors, Object-oriented software composition, pages 99 - 121. Prentice Hall, 1995.

[14] S. Rudkin. Inheritance in LOTOS. In K. Parker and G. Rose, editors, FORTE '92, pages 409-424. Elsevier, 1992.

[15] G. Smith. The Object-Z Specification Language. Kluwer Academic Publisher, 2000.

[16] W.M.P. van der Aalst and T. Basten. Life-cycle inheritance - a Petri-net-based approach. In P. Azema and G. Balbo, editors, $A T P N$ '97, number 1248 in LNCS, pages 62-81. Springer, 1997.

[17] P. Wegner and B. Zdonik. Inheritance as an incremental modification mechanism or What like is and isn't like. In ECOOP'88, volume 322 of LNCS. Springer, 1988.

[18] J. C. P. Woodcock and C. C. Morgan. Refinement of state-based concurrent systems. In VDM Symposium 1990, volume 428 of LNCS, pages 340-351. Springer-Verlag, 1990. 